\title{
Efectos de un programa de entrenamiento del core en el equilibrio de estudiantes de 10 - 12 años
}

\author{
Effects of a training program of core in the balance of 10-12 years students
}

Rocío Mabel Ortega Medina', Sonia Carolina Mantilla Toloza²

Para citar este artículo: Ortega RM, Mantilla SC. Efectos de un programa de entrenamiento del Core en el equilibrio de estudiantes de 10 - 12 años. UstaSalud. 2017;16: 28-34

Licencia Creative Commons

\section{(c) (1) (5) $\Theta$} lo tanto, los lectores pueden acceder libremente a los artículos en su formato .pdf, igualmente podrán descargarlos y difundirlos; sin embargo no podrán modificarlos o alterarlos, adicionalmente se debe reconocer la autoría de las personas que figuran en las publicaciones, pero estas no podrán comercializadas.

'Institución Educativa Provenza, Bucaramanga, Colombia.

${ }^{2}$ Facultad de Salud, Universidad de Pamplona, Pamplona, Colombia.

Autor de correspondencia: Rocío Mabel Ortega Medina Correo electrónico: rosember52@hotmail.com
Recibido para publicación: 10 de octubre de 2016. Aceptado para publicación: 25 de marzo de 2017.

\section{RESUMEN}

Objetivo: Evaluar los efectos de un programa de entrenamiento de la musculatura del core en el equilibrio de los estudiantes, entre 10-12 años, de la Institución Educativa Provenza del municipio de Bucaramanga.

Métodos: Se realizó un estudio cuasi-experimental, con evaluaciones antes y después de una intervención durante 16 semanas, con una frecuencia de tres días por semana y una duración de 60 minutos cada sesión. Esta investigación comprendió una muestra de 40 estudiantes divididos en dos grupos (grupo intervención $n=20$ ) (grupo control $n=20$ ). Se utilizó la prueba de Equilibrio Estático (PEOC 5-30), la prueba de Equilibrio Dinámico (prueba de caminar sobre una barra de equilibrio), el test de decúbito prono, el test de decúbito lateral derecho e izquierdo, el test de flexores de tronco y el test en puente prono para evaluar la resistencia del tronco.

Resultados: Se observó que los dos grupos, tanto el de control como el de intervención mejoraron en todas las pruebas después de una intervención, encontrandose diferencia estadisticamente significativa para dos de las pruebas: Equilibrio estático $(p=0,014)$ y Lateral derecha $(p=0,013)$.

Conclusiones: El principal hallazgo evidenciado fue la mejoría en las variables evaluadas, principalmente en el equilibrio estático y la lateral derecha.

Palabras clave: Educación y entrenamiento físico, fuerza muscular, ejercicio.

\section{ABSTRACT}

Objective: Evaluate the effects of a training program in the core muscles and the balance of students between 10-12 years of a School located in Provenza, Bucaramanga.

Methods: A quasi-experimental study was carried out with a pre and post evaluation after a 16 weeks intervention, the frequency of the intervention was three days a week and every session lasted for 60 minutes. A sample of 40 students was divided into two groups (intervention group $n=20$ ) (control group $n=20$ ). The tests used were: a Static Equilibrium test (PEOC 5-30), Dynamic Balance test (test of walking on a balance beam), Prone test, Right and Left Lateral Decubitus, Trunk Flexion and Prone in Bridge tests for assessing the resistance of trunk.

Results: An improvement after intervention was observed for every test in both groups, statistical significance differences were found in two tests, the static equilibrium test and the right lateral test.

Conclusions: The major finding evidenced was the improvement in the variables assessed mainly the static equilibrium test and the right lateral.

Keywords: Physical education and training, muscle strength, exercise. 


\section{INTRODUCCIÓN}

A medida que el tiempo avanza se han aumentado los riesgos de lesiones físicas en los niños en relación con la actividad física. La investigación europea en poblaciones infantiles ha identificado diferentes factores de riesgo ${ }^{1}$, dentro de ellos las caídas ${ }^{2}$, disminución de la propiocepción y la falta de formación específica del deporte ${ }^{3}$. Teniendo en cuenta lo anterior, lo que respecta a nuestro país y el entorno de nuestras instituciones, es claro que los niños también se enfrentan diariamente a lesiones a causa de diferentes factores. Las consecuencias de estas lesiones pueden dejar secuelas en los niños y pueden ser modificadas o disminuidas con la utilización de programas basados en el desarrollo o mejoramiento de las cualidades físicas dentro de las cuales se encuentra el equilibrio. En un estudio realizado por Larzen et al. ${ }^{4}$ se encontró que la falta de equilibrio aumenta el riesgo de lesiones traumáticas, además se menciona que la falta de fuerzas estabilizadoras (core) (núcleo) están significativamente relacionadas con una lesión de tobillo traumática.

El equilibrio motriz se considera como uno de los aspectos fundamentales de la actividad física de los seres humanos a lo largo de su existencia5, puesto que aporta bases fuertes para el desarrollo de otras capacidades y facilita el control del organismo ante estímulos externos que requieran del control y mantenimiento de una postura, disminuyendo así la prevalencia de estas lesiones.

Otros autores consideran que el equilibrio es la capacidad de mantener el cuerpo en una determinada postura venciendo la acción de la gravedad ${ }^{6}$. Estas definiciones resaltan la importancia del equilibrio en el mantenimiento de las condiciones físicas de un individuo, en la ejecución de actividades diarias, en la prevención de caídas y por supuesto, en la práctica adecuada de actividad física.

El core es un término definido como centro anatómico y funcional del cuerpo, según Bliss y Teeple ${ }^{7}$ hace referencia al complejo muscular situado en la parte central del cuerpo (región lumbo-pélvica) que incluye 29 músculos que estabilizan la columna vertebral y la región abdominal e incluye músculos del abdomen, espalda, parte posterior y anterior de la cadera, suelo pélvico y diafragma ${ }^{8}$. La fuerza del core es una habilidad del complejo neuromuscular lumbo-pélvico que previene las lesiones de la columna vertebral y facilita la capacidad de devolver el equilibrio luego de un estímulo ${ }^{9}$.

Teniendo en cuenta la realidad del país, desde los aspectos de vida saludable y relacionados con la educación física, es necesario hacer una revisión y aplicación a los métodos de trabajo en la parte de equilibrio para escolares, unido al fortalecimiento del core, como instrumento de prevención de lesiones físicas en los niños y adolescentes. De lo anterior, surge la necesidad de idear un programa novedoso, con posturas y actividades que permitan desarrollar y fortalecer las distintas habilidades físicas requeridas por la persona que se encuentra en edad escolar, de manera tal, que potencie las competencias específicas para el niño escolar, en especial, la postura y el equilibrio, para que a su paso al nivel de secundaria, pueda continuar sin problemas de falta de desarrollo de algunas habilidades, el programa planteado para el nivel y edad consideró los lineamientos del Ministerio de Educación Nacional.

Esta investigación tuvo como objetivo estudiar los efectos de un programa de entrenamiento de musculatura core en el equilibrio estático y dinámico de los estudiantes de 10 - 12 años de la Institución Educativa Provenza del municipio de Bucaramanga, con el fin de que sus resultados aporten al diseño de los ejercicios que fundamentan la técnica del core y que unidos a actividades de equilibrio contribuyan al desarrollo y fundamentación de las ciencias de la actividad física.

\section{MATERIALES Y MÉTODOS}

Se realizó un estudio cuasi-experimental con una evaluación antes y después de la intervención. La muestra se seleccionó por conveniencia y estuvo conformada por un grupo control $(n=20)$ que realizó ejercicios basados en trabajo de equilibrio y un grupo de intervención con un programa de entrenamiento de la musculatura del core $(n=20)$. Los participantes fueron estudiantes de 10 a 12 años matriculados en la Institución Educativa Provenza que cursaban quinto grado en el 2013. Los participantes inscritos en una planilla, obtuvieron un código de manera alternada para ser ubicados en los dos grupos, número uno para el grupo control y número dos para el grupo de intervención. Como criterios de exclusión se tuvo en cuenta que los participantes no debían presentar alguna enfermedad diagnostica- 
da que impidiera la realización de actividad física, al igual que individuos con: retraso psicomotor, lesión cerebral, problemas de vértigo, déficits perceptuales y de aprendizaje, alguna deficiencia sensorial, antecedentes de dolor lumbar activo, alteraciones físicas o limitaciones funcionales que impidieran el desarrollo de las actividades.

La variable dependiente y principal por evaluar fue el equilibrio estático y dinámico; como variables independientes se consideró el programa de entrenamiento core, la edad en años, el peso en kilogramos, la estatura en centímetros, el índice de masa corporal mediante la ecuación peso/talla, el estrato socioeconómico, el sexo y la actividad física.

Para la evaluación del equilibrio estático se utilizó la prueba PEOC-5-30 $0^{10}$, que valora el equilibrio estático con un apoyo monopodal y con los ojos cerrados. Para el equilibrio dinámico se utilizó la prueba de caminar sobre una barra de equilibrio ${ }^{11}$, donde el sujeto camina sobre una barra de equilibrio tan rápido como pueda, y se registra el tiempo y la distancia recorrida, este test presenta una fiabilidad del 0,98.

Para la evaluación de la resistencia del core se utilizó el test en decúbito prono, el test en decúbito lateral derecho e izquierdo, el test de resistencia de los flexores de tronco y el test de puente en prono ${ }^{12}$.

La intervención comprendió 16 semanas, en cada una de ellas 3 sesiones, con una duración de $60 \mathrm{mi}-$ nutos, se realizaron tres etapas, una etapa inicial con 8 a 10 ejercicios diferentes de 8 a 10 repeticiones con el aprendizaje y control del movimiento ${ }^{13}$, en la etapa intermedia de 8 a 10 ejercicios de 10 a 12 repeticiones, con una intensidad moderada, y en la etapa final 8 a 10 ejercicios de 12 a 15 repeticiones, con una intensidad moderada ${ }^{14}$ (la cual se tuvo en cuenta por la edad de los niños y de acuerdo con la American College of Sports Medicine (ACSM) y cada sesión constó de cuatro etapas principales: el calentamiento, la flexibilización, el fortalecimiento y la vuelta a la calma.

\section{Análisis estadístico}

Se hizo un análisis descriptivo de las características de los participantes mediante medidas de tendencia central y dispersión. Las variables categóricas se presentan por medio de frecuencias absolutas y relativas; las variables cuantitativas con distribución normal se presentan con media y desviación estándar, aquellas que no presentan distribución normal se describen con mediana y rangos. Las variables fueron comparadas entre los grupos mediante las pruebas de T de Student Pareada y la prueba U. de Mann-Whitney para variables con distribución normal y las que no la presentaban, respectivamente. Para evaluar el efecto de los entrenamientos en el promedio de las puntuaciones finales para cada test se realizó un análisis de covarianza (ANCOVA), siendo la variable dependiente el puntaje final de cada prueba ajustado por el puntaje inicial, el grupo de intervención y la edad. Para todo el análisis se consideró un nivel de significancia $p<0,05$, la base de datos se realizó en el programa Microsoft Excel $^{\circledR}$ (Excel 2013; Microsoft Corporation, Redmont: WA, USA) y el análisis con el paquete estadístico STA$\mathrm{TA}^{\oplus} 11$ (Stata Corp, 2009. Stata Statistical Software: Release II. College Station, TX: Stata Corp LP).

\section{Aspectos éticos y legales}

Esta investigación fue aprobada por el Comité de Investigaciones del Programa de Maestría en Ciencias de la Actividad Física de la Universidad de Pamplona, se tuvieron en cuenta las consideraciones éticas según la Resolución 8430 de 1993, por las cuales este estudio se consideró de riesgo mínimo. Se respetaron los principios éticos de confidencialidad, beneficencia, no maleficencia y justicia. Dentro de las consideraciones éticas se contempló que los estudiantes debían contar con un consentimiento informado para participar en el estudio firmado por sus padres y dar su asentimiento de manera verbal.

\section{RESULTADOS}

La población evaluada estuvo conformada por 22 (55\%) niñas y 18 niños (45\%), no hubo diferencia estadísticamente significativa entre los grupos del estudio respecto al género $(\mathrm{p}=0,204)$, ni tampoco se encontró entre las características antropométricas el nivel de actividad física y el estrato socio económico; sin embargo, la edad de los grupos en la línea de base sí presentó diferencia estadísticamente significativa $(\mathrm{p}=0,024)($ Tabla 1$)$. 
Tabla 1. Características de la población

\begin{tabular}{|c|c|c|c|c|}
\hline Variable & $\begin{array}{c}\text { Grupo de } \\
\text { intervención } \\
n=20\end{array}$ & $\begin{array}{c}\text { Grupo } \\
\text { control } \\
\mathrm{n}=20\end{array}$ & $\begin{array}{l}\text { Total } \\
\mathrm{n}=40\end{array}$ & $\mathrm{p}$ \\
\hline Edad (X \pm D.E. $)$ & $10,3 \pm 0,73$ & $11 \pm 1,0$ & $10,6 \pm 0,93$ & $0,024^{*}$ \\
\hline Peso en kg (X \pm D.E. $)$ & $34,6 \pm 1,20$ & $35,3 \pm 1,11$ & $34,9 \pm 5,13$ & $0,639^{*}$ \\
\hline Estatura en $\mathrm{m}(\mathrm{X} \pm$ D.E. $)$ & $1,4 \pm 0,02$ & $1,5 \pm 0,02$ & $1,4 \pm 0,1$ & $0,222^{*}$ \\
\hline IMC (X \pm D.E. $)$ & $17,6 \pm 0,62$ & $17 \pm 0,51$ & $17,3 \pm 0,4$ & $0,456^{*}$ \\
\hline \multicolumn{5}{|l|}{ Género n (\%) } \\
\hline Masculino & $11(61)$ & $7(39)$ & $18(55)$ & $0,204^{* *}$ \\
\hline Femenino & $9(41)$ & $13(59)$ & $22(45)$ & \\
\hline \multicolumn{5}{|l|}{ Actividad física n (\%) } \\
\hline No & $15(45,5)$ & $18(54,5)$ & $33(82,5)$ & $0,212^{* *}$ \\
\hline Sí & $2(28,6)$ & $5(71,4)$ & $7(17,5)$ & \\
\hline \multicolumn{5}{|c|}{ Estrato socioeconómico n (\%) } \\
\hline 1 & $7(43,7)$ & $9(56,3)$ & $16(40)$ & $0,887^{* *}$ \\
\hline 2 & $9(53)$ & $8(47)$ & $17(42,5)$ & \\
\hline 3 & $2(67)$ & $1(33)$ & $3(7,5)$ & \\
\hline 4 & $2(50)$ & $2(50)$ & $4(10 \%)$ & \\
\hline
\end{tabular}

$\mathrm{X} \pm$ D.E.: promedio \pm desviación estándar, ${ }^{*}$ test de student, ${ }^{* *} \mathrm{Chi}^{2}$.

En cuanto a los resultados antes y después de la intervención se observa que los dos grupos, tanto el de control como el de intervención mejoraron en todas las pruebas después de las 16 semanas, particularmente se evidencian mejores resultados para las pruebas: equilibrio estático y lateral derecha (Tabla 2).

Se puede observar también que los dos grupos de niños mejoraron sus resultados con relación a la eva- luación inicial. Sin embargo, al comparar el efecto entre los dos grupos intervenidos, el análisis de covarianza encontró que la diferencia entre los dos entrenamientos solo fue significativa para dos de las pruebas: equilibrio estático y lateral derecha. Adicionalmente, se puede observar un aumento estadísticamente significativo en el tiempo en segundos en los participantes que hacían parte del grupo de core de 3,9 [IC 95\%: 0,854 - 6,987] y en la prueba de lateral derecha en los mismos participantes con un aumento en el promedio de 8,7 segundos [IC 95\%: 1,96915,417] (Tabla 3).

Debido a que en el análisis bivariado se encontró una diferencia significativa entre los promedios de edad de los grupos, se realizó el ajuste por edad y el valor de $p$ no fue significativo en ninguna de las pruebas.

Tabla 3. Análisis del efecto de las intervenciones entre el grupo control y el de entrenamiento del core ajustado por el puntaje inicial (análisis de Covarianza ANCOVA).

\begin{tabular}{lccc}
\hline \multicolumn{1}{c}{ Test } & Delta & p & [IC 95\%] \\
\hline Equilibrio estático & 3,921 & $0,014^{*}$ & {$[0,854-6,987]$} \\
Equilibrio dinámico & 1,435 & 0,396 & {$[-1,557-4,822]$} \\
\hline Extensión de tronco & 15,730 & 0,081 & {$[-2,040-33,496]$} \\
\hline Lateral derecha & 8,692 & $0,013^{*}$ & {$[1,969-15,417]$} \\
\hline Lateral izquierda & 1,647 & 0,824 & {$[-13,267-16,560]$} \\
Flexión de Tronco & 9,058 & 0,162 & {$[-3,796-21,914]$} \\
\hline Puente prono & $-0,397$ & 0,913 & {$[-7,720-6,926]$} \\
Total & $\mathbf{5 , 3 5 3}$ & $\mathbf{0 , 8 6 1}$ & {$[-56,316-\mathbf{6 7 , 0 2 6}]$} \\
\hline
\end{tabular}

IC: Intervalo de confianza del 95\%.

Tabla 2. Pruebas de equilibrio estático y dinámico pre y post intervención

\begin{tabular}{lcccccc}
\hline \multicolumn{1}{c}{ Test } & & Grupo control & & \multicolumn{3}{c}{ Grupo de intervención } \\
& Prueba inicial & Prueba final & p & Prueba inicial & Prueba final & p \\
\hline Equilibrio estático & $16,0 \pm 7,7$ & $16,8 \pm 5,7$ & 0,553 & $13,4 \pm 8,1$ & $19,4 \pm 6,7$ & $<0,001$ \\
\hline Equilibrio dinámico & $12,3 \pm 4,6$ & $17,3 \pm 7,1$ & $<0,001$ & $15,2 \pm 9,9$ & $21,0 \pm 8,5$ & $<0,001$ \\
Extensión de tronco & $41(3-111)$ & $39(1,2-156)$ & $<0,001^{*}$ & $35,2(5-109)$ & $51,3(25-146)$ & $<0,001^{*}$ \\
\hline Lateral derecha & $24,5(2-52,4)$ & $27,9(4-61)$ & $0,161^{*}$ & $14,3(0-88)$ & $29,3(9,2-94)$ & $<0,001^{*}$ \\
Lateral izquierda & $16(1-57,5)$ & $29,4(1-125)$ & $0,018^{*}$ & $30(2-56)$ & $38,3(15,4-77)$ & $<0,001^{*}$ \\
\hline Flexión de tronco & $38(2-130)$ & $42(10-119)$ & $0,121^{*}$ & $27(8-112)$ & $36,2(17-149)$ & $<0,001^{*}$ \\
\hline Puente prono & $32(1,2-97)$ & $36,6(12-103)$ & $0,009^{*}$ & $36,5(10-120)$ & $40,2(12-135)$ & $0,011^{*}$ \\
\hline Total & $\mathbf{9 8 ( 0 - 3 5 0 )}$ & $\mathbf{1 3 2 , 4 ( 1 - 3 8 4 )}$ & $\mathbf{0 , 1 6 7 ^ { * }}$ & $\mathbf{1 0 3 ( 0 - 2 6 9 )}$ & $\mathbf{1 0 8}(\mathbf{2 5 - 5 2 6 )}$ & $\mathbf{0 , 0 5 2 ^ { * }}$ \\
\hline
\end{tabular}

Valores expresados como media y \pm : desviación estándar o como mediana y (rango), T de Student Pareada, ${ }^{*}$ Prueba U de Mann-Whitney. 


\section{DISCUSIÓN}

Este estudio es de los primeros que busca investigar los efectos del entrenamiento del core en escolares de 10 a 12 años de edad, lo que implica un aporte a la investigación científica, porque abre la puerta y da las bases en la realización de nuevos estudios relacionados con el tema. Se demostró que tanto para el grupo control como para el grupo de intervención, hubo mejoría en el equilibrio estático y dinámico en todas las pruebas después de 16 semanas; los mejores resultados se obtuvieron en las pruebas de equilibrio estático y lateral derecha para los individuos evaluados. En el análisis multivariado, la edad no tuvo repercusión en el efecto del entrenamiento entre los grupos, demostrando de esta manera que no actúa como una variable confusora en el estudio.

En el estudio de Allen et al. ${ }^{15}$ se investigaron los efectos de un trabajo de acondicionamiento básico rutinario en las pruebas de tronco y resistencia muscular del core en niños en edad escolar, aplicando una intervención que se realizó una vez por semana, durante un período de 6 semanas en el inicio de las clases de educación física; ellos encontraron que los tamaños del efecto ( $\mathrm{T}$ de Cohen) fueron 0,61 para la prueba de lumbar en silla romana, 0,44 para el puente prono, 0,27 para el lateral, 0,67 para la dinámica Curl-up, y 0,69 para el estática Curl-up. Estos resultados indicaron que la intervención tuvo un efecto moderado para la mayoría de las pruebas. El análisis reveló coeficientes significativos $(\mathrm{p}=0,001)$ para cada elemento de prueba de control de género, nivel de grado, y las correlaciones pos- test / pre. Los estudiantes en edad escolar media tuvieron mayores aumentos en cuanto al rendimiento en la prueba de la aptitud de pre- a post- test en comparación con los estudiantes en edad escolar primaria. Al comparar estos resultados, con los del presente estudio, se aprecia que fueron utilizadas tres de las mismas pruebas, y se observan mejorías en ellas, pero como conclusión al comparar edades ente 9 y 14 años, se puede pensar que entre más aumenta la edad se observan mejores resultados en la resistencia muscular, lo cual se puede utilizar como herramienta en las clases de educación física, especialmente después de los 10 años de edad, porque en ellos tiene un mayor efecto.

Granacher et al. ${ }^{16}$ investigaron los efectos del entrenamiento en los músculos del core en superficies estables en comparación con superficies inestables, sobre la aptitud física en los niños en edad escolar. Las pruebas utilizadas fueron Emery prueba de equilibrio, prueba de equilibrio Y, prueba de stand and reach, $20 \mathrm{~cm}$ de velocidad, prueba de salto longitudinal y prueba de Bourban TMS. Los resultados del estudio evidenciaron que no se observaron diferencias significativas en cuanto al tiempo (pre vs pos) para el TMS $(8-22 \%, f=0,47-0,76)$, el salto hacia los lados de ensayo $(4-5 \%, \mathrm{f}=1,07)$, y la prueba de equilibrio $\mathrm{Y}(2-3 \%$, $\mathrm{f}=0,46-0,49)$.

Este estudio demuestra los resultados que tiene el trabajo de la musculatura core en el equilibrio, pero no se observan cambios significativos entre bases estables e inestables, lo que se debería considerar para futuros estudios que busquen el diseño y la implementación de los programas que se aplican.

En un estudio reportado por Sandrey y $\mathrm{Mitzel}^{17}$ se investigaron efectos de un programa básico de entrenamiento de la musculatura del core, en el equilibrio dinámico y la fuerza, para los atletas de pista y campo en escuela secundaria. Las sesiones de entrenamiento fueron de 30 minutos en cada sesión y 3 veces por semana; los resultados del análisis mostraron que se mejoraron significativamente las 3 direcciones del SEBT (prueba de balance en excursión de estrella) PM (posteromedial), M (media) y AM (anteromedial), AFT (prueba de abdominalfatigue), BET (prueba de back extensor), SBT (prueba de lado puente) para el lado derecho e izquierdo. El tamaño del efecto fue grande para todas las variables excepto para PM (posteromedial) y AM (anteromedial), donde se observó un efecto moderado. Este estudio utilizó pruebas aplicadas al deporte en el equilibrio, se diferencia puesto que los resultados fueron notables en el equilibrio dinámico debido a que las actividades y entrenamientos fueron encaminadas en bases inestables.

Los resultados del estudio señalan que los dos entrenamientos propuestos, tanto para el grupo de intervención como para el grupo control, mejoran el equilibrio en los participantes, evidenciado en el aumento de los puntajes de las pruebas antes y después de la intervención y estadísticamente verificados. Adicionalmente, el efecto del entrenamiento del core fue más eficaz en el mejoramiento del equilibrio estático y lateral derecha. En este sentido, estos hallazgos se 
relacionan con los del estudio realizado por Iacono et al. ${ }^{18}$ en el 2014, en el que se investigaron los efectos del equilibrio estático y dinámico, con un programa de la musculatura del core durante cuatro semanas en veinte jugadores de fútbol masculino. Para evaluar el equilibrio se utilizó la prueba de excursión; al analizar los resultados de la varianza indican que no hay diferencia significativa en las características antropométricas de los atletas. Los factores correlacionados fueron confiables, con coeficientes de [0,80-0,99]. Los resultados de la varianza antes y después del entrenamiento y las múltiples pruebas comparadas demuestran cambios en los resultados. La puntuación compuesta de la Sebt (prueba de excursión en estrella) mejoró de $107,9 \pm 10,3 \%$ a $114,2 \pm 9,1 \%$ de la longitud de la extremidad inferior derecha $(\mathrm{p}=0,04)$ siguiendo el programa de entrenamiento de la musculatura core; por el contrario no hubo ningún cambio significativo $(\mathrm{p}=0,75)$ en el puntaje de la prueba Sebt compuesto, por la derecha $(99,2 \pm 12,5 \%$ de $99.2 \pm 12,6 \%)$ y la extremidad izquierda (de $101,5 \pm 9,9 \%$ a $104,5 \pm 13,1 \%$ ) en el grupo control $(p=0,06)$. Al analizar los cambios y las diferencias entre los individuos de cada grupo (IG Vs CG) por el entrenamiento (antes y después del entrenamiento) no se observan cambios significativos en el control de la lateralidad y del equilibrio dinámico.

Este último hallazgo en esta investigación se asemeja a los resultados encontrados en este estudio, observación que se analiza teniendo en cuenta que el programa de entrenamiento de core utilizado fue más dirigido en actividades y posturas estáticas, lo que lleva a pensar que este tipo de programas enfatizan especialmente el equilibrio estático, razón por la cual se deja a un lado o no muestra resultados destacables en el equilibrio dinámico.

En el presente trabajo no se realizó enmascaramiento de los evaluadores, entrenadores o participantes, ya que la investigadora fue quien acompañó los entrenamientos, realizó la evaluación de los participantes y el monitoreo de los resultados; se sugiere, sin embargo, considerar un cegamiento para futuras investigaciones. Otro de los limitantes de esta investigación fue la utilización de métodos de evaluación indirectos que no permitían observar los cambios en el músculo específicamente, comparada con otras herramientas como la EMG (electromiografía) de superficie o dinamometría que resultan ser mucho más pre- cisas. Adicionalmente, se reconoce también que para algunas intervenciones de entrenamiento del core el uso especializado de equipamiento para la musculatura del tronco y el core, muchas personas, especialmente los niños, pueden no tener acceso al material especializado. En las clases de educación física o durante la práctica deportiva, puede ser difícil tener un equipo especializado que ayude en el trabajo de la musculatura del core.

Debido al tamaño de la muestra, los resultados de este trabajo solo pueden generalizarse en niños con características similares a las del presente estudio, la extrapolación de los resultados debe realizarse de manera pertinente. El tiempo de aplicación fue de 16 semanas, lo que permite un entrenamiento prolongado, con lo cual se hacen procesos más afines con la observación del trabajo realizado en cada uno de los participantes, contrario a lo que utilizan la gran mayoría de estudios, tiempos menores en la intervención. En futuros trabajos se recomienda seleccionar aleatoriamente a los participantes, realizar enmascaramiento y aumento del tamaño de la muestra. En un futuro trabajo se podría tener en cuenta un grupo control sin intervención, para poder determinar la eficacia del entrenamiento convencional y del core frente a los niños que no realizan ningún ejercicio.

Este trabajo es el primero realizado en la región acerca del efecto del entrenamiento del core en escolares entre 10 y 12 años, por ello esta investigación se convierte en una base para otros estudios, permitiendo la ampliación de técnicas y métodos que aporten al desarrollo del quehacer como docente y profesionales de actividad física en diferentes poblaciones, así como a futuro poder obtener resultados óptimos que enriquezcan el campo de acción y el cumplimiento de metas de mayor magnitud.

\section{REFERENCIAS}

1. Hedström EM, Bergström U, Michno P. Injuries in children and adolescents-Analysis of 41,330 injury related visits to an emergency department in northern Sweden. Injury. 2012;43(9):1403-8. doi: 10.1016/j. injury.2011.01.027.

2. Emery CA. Risk factors for injury in child and adolescent sport: a systematic review of the literature. Clinical Journal of Sport Medicine. 2003;13(4):256-68. 
3. Schneider S, Yamamoto S, Weidmann C, Brühmann B. Sports injuries among adolescents: Incidence, causes and consequences. J Paediatr Child Health. 2012;48(10):E1839. doi: $10.1111 /$ j.1440-1754.2012.02578.x.

4. Larsen LR, Kristensen PL, Junge T, Fuglkjær Møller S, Juul-Kristensen B, Wedderkopp N. Motor performance as risk factor for lower extremity injuries in children. Medicine \& Science in Sports \& Exercise. 2016;48(6):113643. doi: 10.1249/MSS.0000000000000877.

5. Cabedo-Sanromà J, Roca-Balasch J. Evolución del equilibrio estático y dinámico desde los 4 hasta los 74 años. Apuntes. Educación Física y Deportes. 2008;92(2):15-25.

6. Filippetto MC. Acondicionamiento físico acuático y estimulación neuromuscular mecánica: efectos en el equilibrio, la fuerza y la flexibilidad en mujeres de edad adulta intermedia [Trabajo de Grado]. Lleida: Universitat de Lleida. 2012.

7. Bliss LS, Teeple P. Core stability: the centerpiece of any training program. Current sports medicine reports. 2005;4(3):179-83. doi: 10.1097/01. CSMR.0000306203.26444.4e.

8. Willardson JM. Core stability training: applications to sports conditioning programs. J Strength Cond Res. 2007;21:979-85. doi: 10.1519/R-20255.1.

9. Leetun DT, Ireland ML, Willson JD, Ballantyne BT, Davis IM. Core stability measures as risk factors for lower extremity injury in athletes. Med Sci Sports Exerc. 2004;36(6):926-934.

10. Forcades J, Galdón O, Valenzuela A. Fiabilidad de la prueba de equilibrio con ojos cerrados PEOC 5-30 en alumnos de primer ciclo de la ESO. Apunts. Educación física y deportes. 2001;4(66):60-2.

11. Martínez E. Pruebas de aptitud física. Barcelona: Editorial Paidotripo, 2002.
12. Chulvi I, Heredia J, Pomar R, Ramón M. Evaluación de la fuerza para la salud: reflexiones para su aplicación en programas de acondicionamiento físico saludable [Internet]. Valencia: PubliCE Standard.Pid; [consultado 2015 Jun 15] 2007. Disponible en: http://www.sobreentrenamiento.com/PubliCE/Articulo.asp?ida=778.

13. American College of Sports Medicine. Exercise prescription. ACSM's Guidelines for Exercise Testing and Prescription. 8th ed. Philadelphia: Wolters Kluwer, Lippincott Williams \& Wilkins, 2010.

14. Colado J, Chulvi I. Criterios para la planificación y el desarrollo de programas de acondicionamiento muscular en el ámbito de la salud. Rodríguez PL. Ejercicio físico en salas de acondicionamiento muscular. Bases científico-médicas para una práctica segura y saludable. Madrid: Editorial Médica Panamericana. 2008;91-127.

15. Allen B, Hannon J, Burns B, Williams P. Effect of a core conditioning intervention on tests of trunk muscular endurance in school-aged children. J Strength Cond Res. 2014;28(7): 2063-70. doi: 10.1519/ JSC.0000000000000352.

16. Granacher U, Schellbach J, Klein K, Prieske O, Baeyens J, Muehlbauer T. Effects of core strength training using stable versus unstable surfaces on physical fitness in adolescents: a randomized controlled trial. BMC Sports Sci Med Rehabilv. 2014;6(1):40. doi: 10.1186/2052-1847-6-40.

17. Sandrey M, Mitzel J. Improvement in dynamic balance and core endurance after a 6-week core-stability-training program in high school track and field athletes. J Sport Rehabil. 2013;22(4):264-71.

18. Iacono A, Martone D, Alfieri A, Ayalon M, Buono P. Core Stability Training Program (CSTP) effects on static and dynamic balance abilities. Gazzetta Medica Italiana. 2014;173(4):197-206. 